\title{
Transition of metabolic phenotypes and risk of subclinical atherosclerosis according to BMI: a prospective study
}

\author{
Lin Lin ${ }^{1,2,3} \cdot$ Jie Zhang ${ }^{1,2,3} \cdot$ Lei Jiang ${ }^{1,3} \cdot$ Rui Du ${ }^{1,3} \cdot$ Chunyan Hu ${ }^{1,3} \cdot$ Jieli Lu ${ }^{1,3} \cdot$ Tiange Wang ${ }^{1,3} \cdot$ Mian $\mathrm{Li}^{1,3}$. \\ Zhiyun Zhao ${ }^{1,3} \cdot \mathrm{Yu} \mathrm{Xu}^{1,3} \cdot$ Min Xu $\mathrm{Xu}^{1,3} \cdot$ Yufang $\mathrm{Bi}^{1,3} \cdot$ Guang Ning ${ }^{1,3} \cdot$ Weiqing Wang ${ }^{1,3} \cdot$ Yuhong Chen ${ }^{1,2,3}$ (D)
}

Received: 8 August 2019 / Accepted: 31 January 2020 / Published online: 4 March 2020

(C) Springer-Verlag GmbH Germany, part of Springer Nature 2020

\begin{abstract}
Aims/hypothesis The cardiometabolic risk associated with metabolically healthy obesity (MHO) remains the subject of debate. It is unclear whether MHO is a transient condition that affects subclinical atherosclerosis risk. In this study, we aimed to investigate the association of $\mathrm{MHO}$ and its transition over time with incident subclinical atherosclerosis.

Methods A prospective study was conducted with 6220 Chinese adults who were free of cardiovascular disease (CVD) at baseline. Obesity was defined as BMI $\geq 25.0 \mathrm{~kg} / \mathrm{m}^{2}$. Metabolic health was defined as an individual having fewer than two of the National Cholesterol Education Program Expert Panel on Detection, Evaluation, and Treatment of High Blood Cholesterol in Adults (NCEP ATP III) criteria for components of the metabolic syndrome (excluding waist circumference). Subclinical atherosclerosis was measured by brachial-ankle pulse wave velocity, pulse pressure and albuminuria, separately or combined. Participants were cross-classified by BMI categories and by metabolic health status and its transition during follow-up. Inverse probability weighted logistic regression models were used to estimate ORs and 95\% CIs for subclinical atherosclerosis. Results The MHO phenotype accounted for $16.3 \%$ of the total population and $32.8 \%$ of the population with obesity at baseline. Baseline MHO was not significantly associated with incident subclinical atherosclerosis. During a follow-up period of 4.4 years, $46.8 \%$ of individuals with MHO developed a metabolically unhealthy status. Those with transient MHO had an increased risk of composite subclinical atherosclerosis compared with those in the metabolically healthy non-obesity reference group (OR 2.52 [95\% CI 1.89, 3.36]). A transition from metabolically unhealthy to healthy status was shown to decrease the outcome risk. The relationship between BMI and subclinical atherosclerosis was partly mediated by BP and plasma glucose.

Conclusions/interpretation MHO is not a stable condition and transient MHO conferred an increased risk of subclinical atherosclerosis, the early stage of CVD. Hence, individuals may benefit from early behavioural or medical management in order to avoid a deterioration of metabolic status and prevent atherosclerosis and CVD.
\end{abstract}

Keywords Metabolic health $\cdot$ Obesity $\cdot$ Prospective study $\cdot$ Subclinical atherosclerosis

Lin Lin, Jie Zhang and Lei Jiang contributed equally to the article.

Electronic supplementary material The online version of this article (https://doi.org/10.1007/s00125-020-05116-5) contains peer-reviewed but unedited supplementary material, which is available to authorised users.

Guang Ning

gning@sibs.ac.cn

Weiqing Wang

wqingw61@163.com

Yuhong Chen

chenyh70@126.com

1 Shanghai Institute of Endocrine and Metabolic Diseases, Department of Endocrine and Metabolic Diseases, Rui-Jin Hospital, Shanghai Jiao Tong University School of Medicine, 197 Rui-Jin 2nd Road, Shanghai 200025, China

2 Department of Endocrine and Metabolic Diseases Rui-Jin Hospital North, Shanghai Jiao Tong University School of Medicine, Shanghai, China

3 State Key Laboratory of Medical Genomics, Key Laboratory for Endocrine and Metabolic Diseases of Ministry of Health, National Clinical Research Center for Metabolic Diseases, Rui-Jin Hospital, Shanghai Jiao Tong University School of Medicine, Shanghai, China 


\section{Research in context}

\section{What is already known about this subject?}

- Obesity with clinically benign metabolic features, referred to as metabolically healthy obesity (MHO), has generally been expected to have a favourable clinical course

- Studies investigating the relationship between $\mathrm{MHO}$ and cardiovascular disease (CVD) by examining MHO at baseline with longitudinal follow-up have produced inconsistent results

- No significant association between MHO and prevalent subclinical CVD was found in the Framingham Heart Study, and the subclinical atherosclerosis risk for $\mathrm{MHO}$ and its transition is still incompletely delineated

What is the key question?

- Can the MHO phenotype at baseline increase subclinical atherosclerosis risk with longitudinal follow-up?

What are the new findings?

- $\mathrm{MHO}$ at baseline was not associated with an increased risk of subclinical atherosclerosis

- Approximately half of the individuals with MHO developed a metabolically unhealthy status within the follow-up period and subsequently exhibited a higher risk of subclinical atherosclerosis, compared with the non-obese reference group

- The association between BMI and subclinical atherosclerosis was partly mediated by metabolic profiles

How might this impact on clinical practice in the foreseeable future?

- Our results provide new evidence that persistent $\mathrm{MHO}$ can be considered as a therapeutic target in the prevention of atherosclerosis and CVD; early behavioural and medical management to prevent the deterioration of metabolic status, or to reverse existing mild metabolic disorders, may prove beneficial

\begin{tabular}{ll}
\multicolumn{2}{l}{ Abbreviations } \\
baPWV & Brachial-ankle pulse wave velocity \\
CVD & Cardiovascular disease \\
DBP & Diastolic BP \\
FPG & Fasting plasma glucose \\
HDL-C & HDL-cholesterol \\
IPW & Inverse probability weighting \\
LDL-C & LDL-cholesterol \\
MESA & Multi-Ethnic Study of Atherosclerosis \\
MHNO & Metabolically healthy non-obesity \\
MHO & Metabolically healthy obesity \\
MUNO & Metabolically unhealthy non-obesity \\
MUO & Metabolically unhealthy obesity \\
PP & Pulse pressure \\
SBP & Systolic BP \\
UACR & Urinary albumin/creatinine ratio
\end{tabular}

\section{Introduction}

Obesity is a worldwide epidemic that imposes a considerable burden on global health; its complications, such as hyperglycaemia, hypertension and hyperlipidaemia, contribute to an increased risk of cardiovascular disease (CVD) and their prevention and treatment have considerable cost implications $[1,2]$. It is of great interest to find a specific subset of the obese phenotype that is resilient to obesity-associated cardiovascular outcomes, thus allowing us to focus interventions on those likely to benefit most and to formulate personalised treatment. In effect, there is a unique condition of being obese yet having a relatively favourable metabolic profile, referred to as metabolically healthy obesity (MHO) [3]. The prognostic value of $\mathrm{MHO}$ in the primary prevention of CVD is the subject of an ongoing debate [4]. Given that the onset of clinical CVD events, following the appearance of metabolic risk factors, requires a long period of time [5], the confirmation of subclinical atherosclerosis, manifested by elevated pulse wave velocity, pulse pressure (PP) or albuminuria [6-9], may play an important role in the primary prevention of CVD, and may allow better evaluation of the impact of obesity-related factors on the development of overt CVD within a relatively short period.

Whether or not MHO is an innocuous low-risk condition remains contentious, although a wealth of recent evidence has corroborated that MHO is associated with intermediate cardiovascular risk between metabolically healthy non-obesity (MHNO) and metabolically unhealthy obesity (MUO) [10-12]. The remaining inconsistency may be attributed to the heterogeneity of study populations, the absence of a uniform set of criteria used to define MHO and, above all, the transient nature of the MHO phenotype [13, 14]. MHO is not a stable state and former studies have documented that 
33-52\% of individuals with MHO will transition to a metabolically unhealthy phenotype [13-18]. Subsequently, the Multi-Ethnic Study of Atherosclerosis (MESA) study and the Nurses' Health Study confirmed that a large proportion of individuals with MHO developed a metabolically unhealthy status within a decade and exhibited high CVD risk $[15,17]$. Recently, the Framingham Heart Study provided evidence that MHO, compared with MHNO, was not associated with prevalent subclinical CVD [18]. However, the subclinical atherosclerosis risk regarding $\mathrm{MHO}$ and its transition is still incompletely delineated.

In this study, we aimed to examine the association between metabolic health and subclinical atherosclerosis risk according to BMI categories. We focused on the transition of metabolic health status during follow-up, with its concomitant variance in subclinical atherosclerosis risk. Accordingly, we posed three research hypotheses: (1) The risk of subclinical atherosclerosis corresponding to individuals with $\mathrm{MHO}$ at baseline will be intermediate between those of their MHNO and MUO counterparts; (2) transition of $\mathrm{MHO}$ will explain a significant portion of the variance in subclinical atherosclerosis risk; and (3) the relationship between BMI and subclinical atherosclerosis will be mediated by metabolic profiles.

\section{Methods}

Study population The study population was obtained from an ongoing cohort study, which was conducted in a community-based population in Jiading District, Shanghai, China. A detailed description of the study design, eligibility criteria and sampling has been published previously [19]. Briefly, between March and August 2010, a total of 10,375 registered permanent residents of the local community aged 40 years or above were consecutively recruited into the Jiading study. Each participant underwent a face-to-face interview to complete a questionnaire. Blood and urine samples were collected for biochemical measurements. For the present study, we excluded individuals who reported a history of CVD $(n=$ 850). All eligible participants were invited to attend a follow-up visit between August 2014 and July 2015 and a total of 6302 participants attended. We further excluded 82 participants without follow-up data on metabolic profiles, leaving 6220 for this analysis. Additionally, 1992 participants with subclinical atherosclerosis manifested by elevated brachial-ankle pulse wave velocity (baPWV) at baseline were excluded from the baPWV analyses; 1743 participants with subclinical atherosclerosis manifested by elevated PP and 1083 with albuminuria at baseline were excluded from the respective analyses; and 2762 participants with elevated baPWV, elevated PP or albuminuria at baseline were excluded from analyses for composite subclinical atherosclerosis (Fig. 1).

The study protocol was approved by the Committee on Human Research at Rui-Jin Hospital, Shanghai Jiao Tong University School of Medicine. All participants provided written informed consent.

Baseline data collection Face-to-face interviews were performed at baseline and in the follow-up period by trained study personnel using a standard detailed questionnaire to collect information on sociodemographic characteristics, lifestyle factors, medical history and current use of medications. Information on intensity, duration and frequency of physical activity was obtained using the International Physical Activity Questionnaire (IPAQ) [20]. Current smokers or drinkers were defined as those who had smoked cigarettes or consumed alcohol regularly in the past 6 months.

Anthropometric measurements, including body weight, height, waist circumference and BP, were performed by trained study nurses according to standard protocols. BMI was calculated by dividing weight $(\mathrm{kg})$ by height $(\mathrm{m})$ squared. Waist circumference was determined using a measuring tape positioned midway between the lowest rib and the superior border of the iliac crest as the participant exhaled normally. The BP measurement was performed on the non-dominant arm, using an automated electronic device (Omron model HEM-752 FUZZY; Omron Company, Dalian, China). Three BP measurements were taken with participants in a seated position after $5 \mathrm{~min}$ of quiet rest and the mean value of three measurements was used in analysis. PP was defined as systolic BP (SBP) minus diastolic BP (DBP) from the mean of the three measurements.

All the participants underwent baPWV measurements, with baPWV values determined by Colin VP-1000 (model BP203RPEII, form PWV/ABI; Omron Colin Medical Instruments, Tokyo, Japan). Briefly, participants attached cuffs around both arms and ankles after having rested for $10-15 \mathrm{~min}$ at room temperature $\left(25^{\circ} \mathrm{C}\right)$. Measurements from the brachial and tibial arteries were obtained simultaneously. Transit time, the time interval between the initial increase in brachial and tibial waveforms, and transit distance between the arm and ankle were measured. The value of baPWV was calculated as the transit distance divided by the transit time. We adopted the mean value of the right and left common baPWV for analysis at both baseline and follow-up.

All the participants were required to fast for $\geq 10 \mathrm{~h}$ before their visits and underwent a 75 g OGTT. Plasma glucose was measured using the glucose oxidase method on an autoanalyser (Modular P800; Roche, Basel, Switzerland). Total cholesterol, LDL-cholesterol (LDL-C), HDL-cholesterol (HDL-C) and triacylglycerols were measured using the chemiluminescence method with an auto-analyser (Modular E170; Roche, Basel, Switzerland). Blood was shipped at 2- 


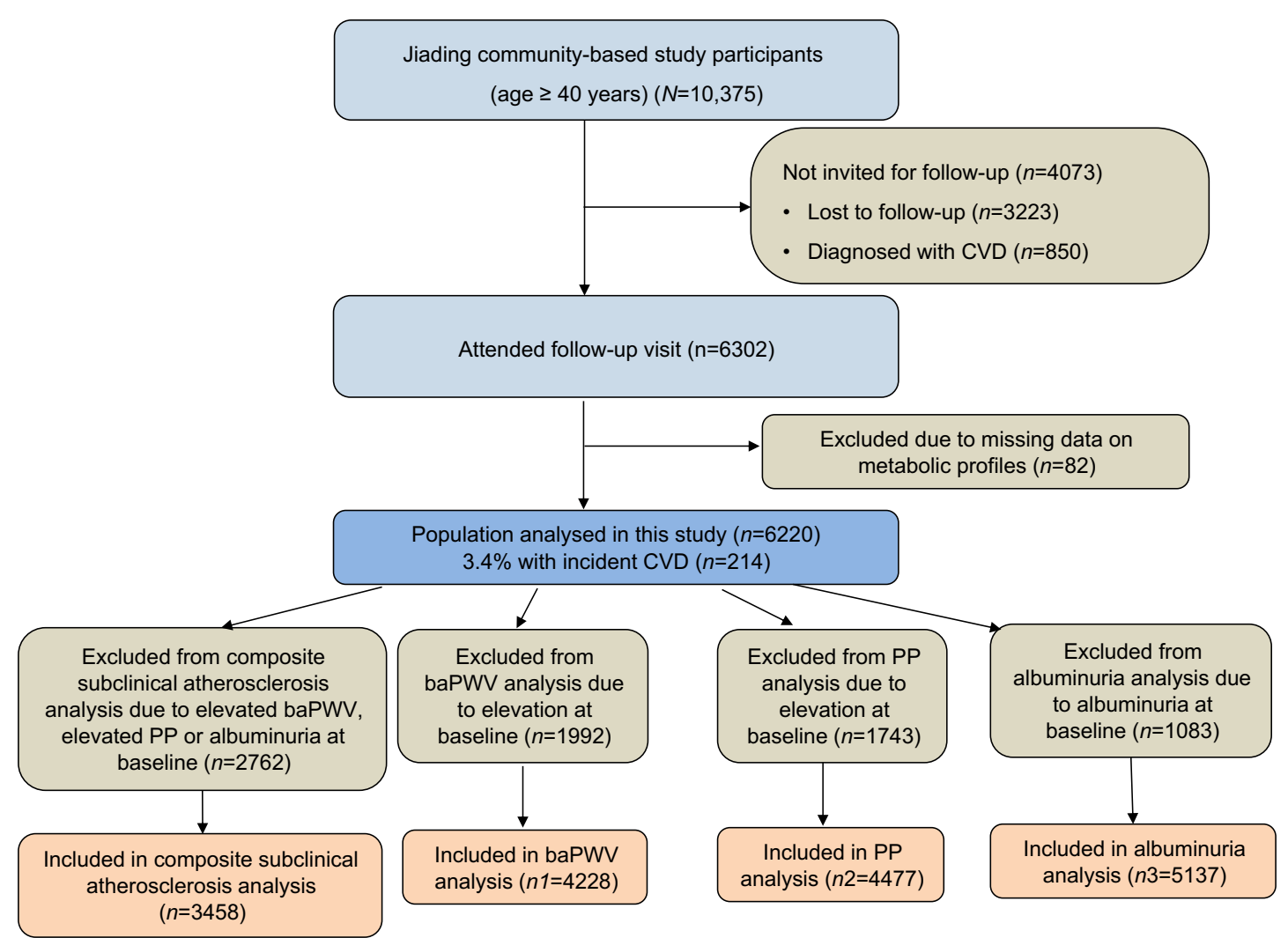

Fig. 1 Flowchart of study population

$8^{\circ} \mathrm{C}$ to a certified central laboratory at Rui-Jin Hospital, Shanghai, China (certificated by the US National Glycohemoglobin Standardization Program and passed the Laboratory Accreditation Program of the College of American Pathologists). A first-voided, early-morning spot urine sample was obtained to measure urinary albumin $(\mathrm{g} / \mathrm{l})$ and creatinine (mmol/l) using an immunoturbidimetric method (Beijing Atom High-Tech, Beijing, China) and Jaffe's kinetic method on an automatic analyser (Hitachi 7600-020, Tokyo, Japan), respectively. Urinary albumin/creatinine ratio (UACR) was calculated by dividing the urinary albumin concentration by the urinary creatinine concentration and is expressed in $\mathrm{mg} / \mathrm{mmol}$. The index of HOMA-IR was calculated as fasting serum insulin $(\mu \mathrm{U} / \mathrm{ml}) \times$ fasting plasma glucose (FPG) (mmol/l)/22.5 [21].

Obesity and metabolic status at baseline We defined the obesity phenotype as BMI $\geq 25 \mathrm{~kg} / \mathrm{m}^{2}$ according to Asiaspecific BMI criteria, which are determined by the WHO Western Pacific Region [22]. The validity of this definition has been confirmed previously [23, 24].

The metabolically healthy state was defined using standard operating protocols to measure Adult Treatment Panel III (ATP III) components. The waist circumference criterion was not used because of its collinearity with BMI. Individuals who had evidence of fewer than two of the following four criteria were considered metabolically healthy: (1) elevated SBP ( $\geq 130 \mathrm{mmHg}$ ) and/or DBP ( $\geq 85 \mathrm{mmHg})$, or on antihypertensive treatment; (2) high triacylglycerols ( $\geq 1.7 \mathrm{mmol} / \mathrm{l}$ ), or on lipid-lowering medications; (3) high FPG ( $\geq 5.6 \mathrm{mmol} / \mathrm{l})$, or on medications for diabetes; and (4) low HDL-C $(<1.04 \mathrm{mmol} / 1$ in men and $<1.29 \mathrm{mmol} / 1$ in women) [25]. Study participants were divided into four groups: (1) MHNO (BMI $<25 \mathrm{~kg} / \mathrm{m}^{2}$ and metabolically healthy status); (2) metabolically unhealthy non-obesity (MUNO; BMI $<25 \mathrm{~kg} / \mathrm{m}^{2}$ and metabolically unhealthy status); (3) MHO (BMI $\geq 25 \mathrm{~kg} / \mathrm{m}^{2}$ and metabolically healthy status); and (4) MUO (BMI $\geq 25 \mathrm{~kg} / \mathrm{m}^{2}$ and metabolically unhealthy status).

Follow-up and outcome assessment From August 2014 to July 2015, all the eligible participants were invited to attend a follow-up examination. The same standard questionnaire as that used at baseline was adopted to collect lifestyle risk factors and medical history. Blood and urine samples were obtained using the same protocols that were used during the baseline examination.

Trained staff used a standard form and made photocopies to collect important data if participants were hospitalised or had visited an emergency department. The information on clinical outcomes was verified by two members of the outcome adjudication committee, with discrepancies being adjudicated 
through discussion within the committee. All members of the committee were unaware of the baseline information of study participants.

The investigated outcomes were elevated baPWV, elevated PP, albuminuria, and their combination as the composite subclinical atherosclerosis. Baseline and incident subclinical atherosclerosis reflected by elevated baPWV was defined as the sex-specific upper quartile of baseline baPWV ( $\geq 1701.0 \mathrm{~cm} / \mathrm{s}$ in men, $\geq 1724.5 \mathrm{~cm} / \mathrm{s}$ in women) $[19,26]$. Baseline and incident subclinical atherosclerosis reflected by elevated PP was defined as the sex-specific upper quartile of baseline PP ( $\geq 65.0 \mathrm{mmHg}$ in men, $\geq 68.2 \mathrm{mmHg}$ in women) [19]. Incident albuminuria was defined as UACR $\geq 3.4 \mathrm{mg} /$ mmol (30 mg/g) [27]. Incident CVD was defined as the first instance of myocardial infarction, stroke, hospitalisation or treatment for heart failure.

Statistical analysis Baseline characteristics were demonstrated according to the combined obesity and metabolic status. Continuous variables were presented as mean \pm SD for the normally distributed variables or median (interquartile range) for the skewed variables. All categorical variables were presented as numbers and proportions. The analyses of continuous and categorical variables to assess differences among the four phenotypes were determined by one-way ANOVA or the $\chi^{2}$ test.

ORs and their corresponding 95\% CIs for combined obesity and metabolic status in relation to incident subclinical atherosclerosis (separate/composite) and CVD were evaluated through multivariate logistic regression analyses. Inverse probability weighting (IPW) was used to address the selection bias. Weights were based on results from a model of follow-up status, estimated using logistic regression with being followed up or not as the dependent variable and the baseline confounding variables, exposure status and causal factors of atherosclerosis (FPG, LDL-C and SBP) as independent variables. We used the multivariable logistic regression analyses, before and after IPW, to assess whether transitioning of metabolically healthy status was associated with higher odds of incident subclinical atherosclerosis (separate/composite). We did a sensitivity analysis with the exclusion of participants who received treatment with glucose-, BP- or lipid-lowering drugs.

We examined potential non-linear associations between the levels of BMI and the incidence of subclinical atherosclerosis (separate/composite) with the use of restricted cubic splines [28]. Analyses were multivariate-adjusted, using three knots, which were located at the 5th, 50th and 95th percentiles for baseline BMI levels, and the highest and lowest $0.5 \%$ were trimmed. Tests for non-linearity, which compared a model containing only the linear term with a model containing the linear and restricted cubic spline terms, were performed using likelihood ratio tests. If a test for non-linearity was not significant, a test for linearity was performed, comparing a model containing the linear term with a model containing only the covariates of interest. Individual mediation analysis was performed to test the effect of metabolic profiles on the association between BMI and composite subclinical atherosclerosis. Indirect effects and $95 \%$ CIs were estimated by bootstrapping with 10,000 resamples.

Significance tests were two-tailed, with a $p$ value $<0.05$ considered as statistically significant. Statistical analysis was performed using SAS version 9.4 (SAS Institute, Cary, NC, USA).

\section{Results}

Baseline sociodemographic and biochemical characteristics of the participants according to metabolic health and obesity status are shown in Table 1. Overall, $54.1 \%(n=3366)$ of the participants were metabolically unhealthy and $49.9 \%$ $(n=3102)$ were obese. The MHO phenotype accounted for $16.3 \%(n=1016)$ of the total population and $32.8 \%$ of the population with obesity. The BP, FPG, triacylglycerols, total cholesterol and LDL-C were higher and HDL-C was lower in metabolically unhealthy individuals, irrespective of obesity. Of note, individuals with MHO and MUNO had BP and plasma glucose and serum lipid levels between those with MHNO and MUO. Estimates for indicators of events, including baPWV, PP and UACR, exhibited a similar pattern.

Figure 2 presents the risk of subclinical atherosclerosis cross-classified by BMI categories and metabolic health. The follow-up period in MHNO, MUNO, MHO and MUO groups was $4.37 \pm 0.25$ years, $4.35 \pm 0.24$ years, $4.39 \pm$ 0.24 years and $4.37 \pm 0.26$ years, respectively. During a median follow-up period of 4.4 years, we documented 842 (24.4\%) cases of subclinical atherosclerosis, $435(8.5 \%)$ cases of albuminuria, $765(18.1 \%)$ cases of subclinical atherosclerosis reflected by elevated baPWV and $583(13.0 \%)$ cases of subclinical atherosclerosis reflected by elevated PP. Individuals with metabolically unhealthy status had an increased risk of subclinical atherosclerosis (separate/composite) compared with the MHNO group (ORs [95\% CIs] 1.73 $[1.25,2.40]$ and $2.08[1.57,2.75]$ for composite subclinical atherosclerosis in MUNO and MUO groups, respectively). The association between $\mathrm{MHO}$ and the risk of subclinical atherosclerosis (separate/composite) was of no significance, although a positive association was apparent.

We next investigated whether the risk of subclinical atherosclerosis was modified by the transition in metabolic health status during follow-up. Overall, $29.2 \%$ of the participants experienced metabolic status changes. BMI decreased during follow-up, except in the individuals who moved from metabolically healthy status to metabolically unhealthy status. Participants who experienced a temporary metabolically healthy status or who reached a stable metabolically unhealthy 
Table 1 Baseline characteristics of participants according to metabolic health and obesity $(n=6220)$

\begin{tabular}{|c|c|c|c|c|c|}
\hline \multirow[t]{2}{*}{ Characteristic } & \multicolumn{2}{|l|}{ Non-obese } & \multicolumn{2}{|l|}{ Obese } & \multirow[t]{2}{*}{$p$ value } \\
\hline & $\begin{array}{l}\text { Metabolically healthy } \\
\text { (MHNO) } \\
(n=1838)\end{array}$ & $\begin{array}{l}\text { Metabolically unhealthy } \\
\text { (MUNO) } \\
(n=1280)\end{array}$ & $\begin{array}{l}\text { Metabolically healthy } \\
(\mathrm{MHO}) \\
(n=1016)\end{array}$ & $\begin{array}{l}\text { Metabolically unhealthy } \\
\text { (MUO) } \\
(n=2086)\end{array}$ & \\
\hline Male sex, $n(\%)$ & $679(36.9)$ & $422(33.0)$ & $426(41.9)$ & $781(37.4)$ & 0.0002 \\
\hline Age, years & $56.94 \pm 9.42$ & $59.22 \pm 9.03$ & $57.90 \pm 8.71$ & $58.34 \pm 8.50$ & 0.0005 \\
\hline BMI, $\mathrm{kg} / \mathrm{m}^{2}$ & $22.34 \pm 1.79$ & $22.98 \pm 1.59$ & $27.23 \pm 1.98$ & $27.92 \pm 2.27$ & $<0.0001$ \\
\hline Waist circumference, $\mathrm{cm}$ & $75.38 \pm 6.37$ & $78.44 \pm 6.09$ & $86.50 \pm 6.72$ & $89.04 \pm 6.83$ & $<0.0001$ \\
\hline $\begin{array}{l}\text { Highschool education or } \\
\text { above, } n(\%)^{\mathrm{a}}\end{array}$ & $446(24.3)$ & $260(20.4)$ & $160(15.8)$ & $351(16.9)$ & $<0.0001$ \\
\hline \multicolumn{6}{|l|}{ Lifestyle factors } \\
\hline Current smokers, $n(\%)^{\mathrm{a}}$ & $405(22.8)$ & $223(18.2)$ & $222(22.6)$ & $423(20.9)$ & 0.51 \\
\hline Current drinkers, $n(\%)^{\mathrm{a}}$ & $187(10.5)$ & $105(8.6)$ & $116(11.7)$ & $213(10.5)$ & 0.52 \\
\hline $\begin{array}{r}\text { Physical activity } \\
\text { (MET-h/week) }\end{array}$ & $15.00(0.00-37.00)$ & $21.00(0.00-38.50)$ & $15.00(0.00-31.50)$ & $18.50(0.00-31.50)$ & 0.003 \\
\hline \multicolumn{6}{|l|}{$\mathrm{BP}, \mathrm{mmHg}$} \\
\hline SBP & $131.09 \pm 18.30$ & $144.68 \pm 17.67$ & $139.33 \pm 19.77$ & $148.79 \pm 18.34$ & $<0.0001$ \\
\hline DBP & $78.24 \pm 9.66$ & $83.94 \pm 9.40$ & $82.83 \pm 10.04$ & $86.58 \pm 10.00$ & $<0.0001$ \\
\hline $\mathrm{PP}$ & $52.86 \pm 14.02$ & $60.74 \pm 15.64$ & $56.50 \pm 15.41$ & $62.21 \pm 16.01$ & $<0.0001$ \\
\hline \multicolumn{6}{|c|}{ Serum lipid concentrations, $\mathrm{mmol} / \mathrm{l}$} \\
\hline Triacylglycerols & $0.99(0.78-1.28)$ & $1.76(1.22-2.31)$ & $1.15(0.92-1.43)$ & $1.90(1.40-2.61)$ & $<0.0001$ \\
\hline Total cholesterol & $5.18 \pm 0.92$ & $5.41 \pm 1.05$ & $5.32 \pm 0.91$ & $5.50 \pm 1.10$ & $<0.0001$ \\
\hline HDL-C & $1.51 \pm 0.31$ & $1.26 \pm 0.32$ & $1.42 \pm 0.27$ & $1.18 \pm 0.27$ & $<0.0001$ \\
\hline LDL-C & $3.03 \pm 0.79$ & $3.23 \pm 0.92$ & $3.22 \pm 0.79$ & $3.31 \pm 0.91$ & $<0.0001$ \\
\hline $\mathrm{FPG}, \mathrm{mmol} / 1$ & $4.97 \pm 0.76$ & $5.96 \pm 1.81$ & $5.00 \pm 0.62$ & $6.08 \pm 1.84$ & $<0.0001$ \\
\hline HOMA-IR & $1.06(0.71-1.47)$ & $1.67(1.14-2.36)$ & $1.44(1.05-2.03)$ & $2.41(1.62-3.57)$ & $<0.0001$ \\
\hline $\mathrm{baPWV}, \mathrm{cm} / \mathrm{s}$ & $1452.11 \pm 295.07$ & $1651.08 \pm 369.01$ & $1477.77 \pm 290.51$ & $1637.69 \pm 334.50$ & $<0.0001$ \\
\hline $\mathrm{UACR}, \mathrm{mg} / \mathrm{mmol}$ & $0.50(0.30-0.90)$ & $0.57(0.32-1.09)$ & $0.51(0.30-0.96)$ & $0.68(0.37-1.42)$ & $<0.0001$ \\
\hline Diabetes, $n(\%)$ & $60(3.3)$ & $335(26.2)$ & $51(5.0)$ & $654(31.4)$ & $<0.0001$ \\
\hline Hypertension, $n(\%)^{\mathrm{a}}$ & $607(33.1)$ & $872(68.1)$ & $516(51.0)$ & $1617(77.5)$ & $<0.0001$ \\
\hline Hypercholesterolaemia, $n(\%)^{\mathrm{a}}$ & $238(13.0)$ & $240(18.8)$ & $155(15.3)$ & $463(22.2)$ & $<0.0001$ \\
\hline \multicolumn{6}{|c|}{ Drug use, $(\%)$} \\
\hline Glucose-lowering drug & $16(0.9)$ & $160(12.5)$ & $14(1.4)$ & $244(11.7)$ & $<0.0001$ \\
\hline BP-lowering drug & $218(11.9)$ & $375(29.3)$ & $221(21.8)$ & $880(42.2)$ & $<0.0001$ \\
\hline Lipid-lowering drug & 0 & $3(0.2)$ & 0 & $8(0.4)$ & 0.01 \\
\hline
\end{tabular}

Data were means \pm SD or medians (interquartile ranges) for skewed variables or numbers (proportions) for categorical variables

$p$ values were calculated from one-way ANOVA for continuous variables and $\chi^{2}$ test for categorical variables

${ }^{a}$ Data are missing for highschool education or above (MHNO, $n=4$; MUNO, $n=7$; MHO, $n=5$; MUO, $n=12$ ), current smokers (MHNO, $n=61$; MUNO, $n=55$; MHO, $n=32$; MUO, $n=64$ ), current drinkers (MHNO, $n=59$; MUNO, $n=52$; MHO, $n=28$; MUO, $n=61$ ), hypertension (MHNO, $n=6$; MHO, $n=4$ ) and hypercholesterolaemia (MHNO, $n=1)$

MET, metabolic equivalent of time

status were more likely to have increasingly worsening metabolic variables, compared with those with stable metabolically healthy status or temporary metabolically unhealthy status (ESM Table 1). Only a small fraction of participants with MUNO or MUO lost their metabolic abnormality during follow-up and they then were not at risk of subclinical atherosclerosis. Of individuals with MHO at baseline, 46.8\% (301 of 643) developed metabolically unhealthy status and they then had an increased risk of composite subclinical atherosclerosis (inverse probability weighted OR [95\% CI] 2.52 [1.89, 3.36]) (Fig. 3). The results for incident subclinical atherosclerosis reflected by elevated baPWV, elevated PP or albuminuria were similar to those for composite subclinical atherosclerosis (Table 2).

As changes in medications taken by participants affected the change in the metabolic health status (ESM Table 2), we did an analysis among participants who were not receiving 
Fig. 2 Risk of incident composite subclinical atherosclerosis (a), elevated baPWV (b), elevated PP (c) and albuminuria (d) according to metabolic health and obesity. ORs (95\% CIs) were adjusted for age, sex, current smoking (yes/ no), current drinking (yes/no), education and physical activity

- Unadjusted

- Multivariate adjusted

a

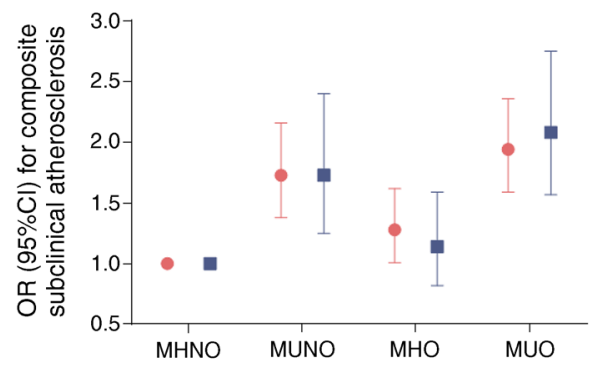

C

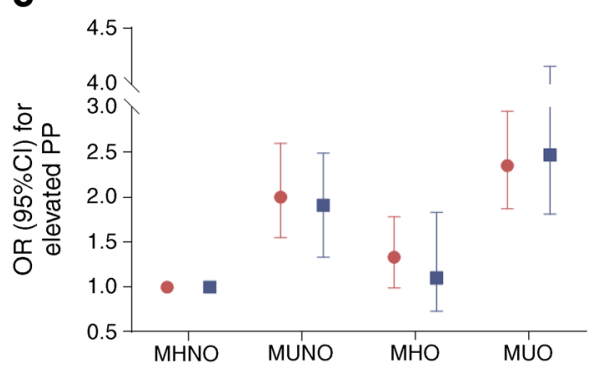

b

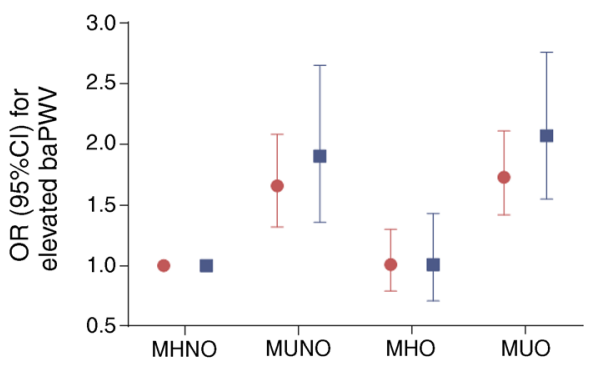

d

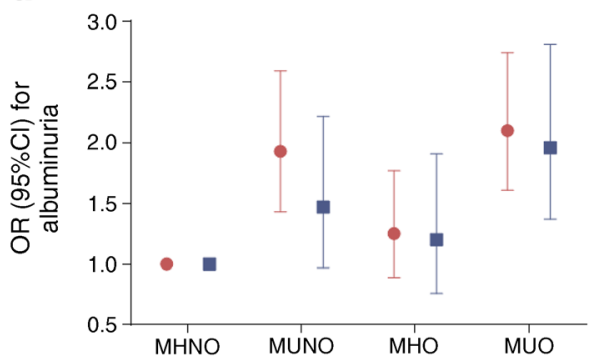

pharmacological treatment. The ORs for all subgroups were generally comparable with those in the main analysis (ESM Table 3). When we investigated the association between metabolic health and incident CVD, the MHO phenotype was not associated with a higher risk of CVD (ESM Table 4). Furthermore, neither stable nor transient MHO phenotype conferred a higher risk of incident CVD (ESM Table 5).
Multivariate-adjusted restricted cubic spline analyses, unadjusted for metabolic profiles, indicated a significant linear association of baseline BMI with subclinical atherosclerosis (Fig. 4). Estimates for the association between baseline BMI and subclinical atherosclerosis risk were strongly attenuated and were non-significant after further adjustment for FPG, triacylglycerol, SBP and HDL-C. Mediation analysis

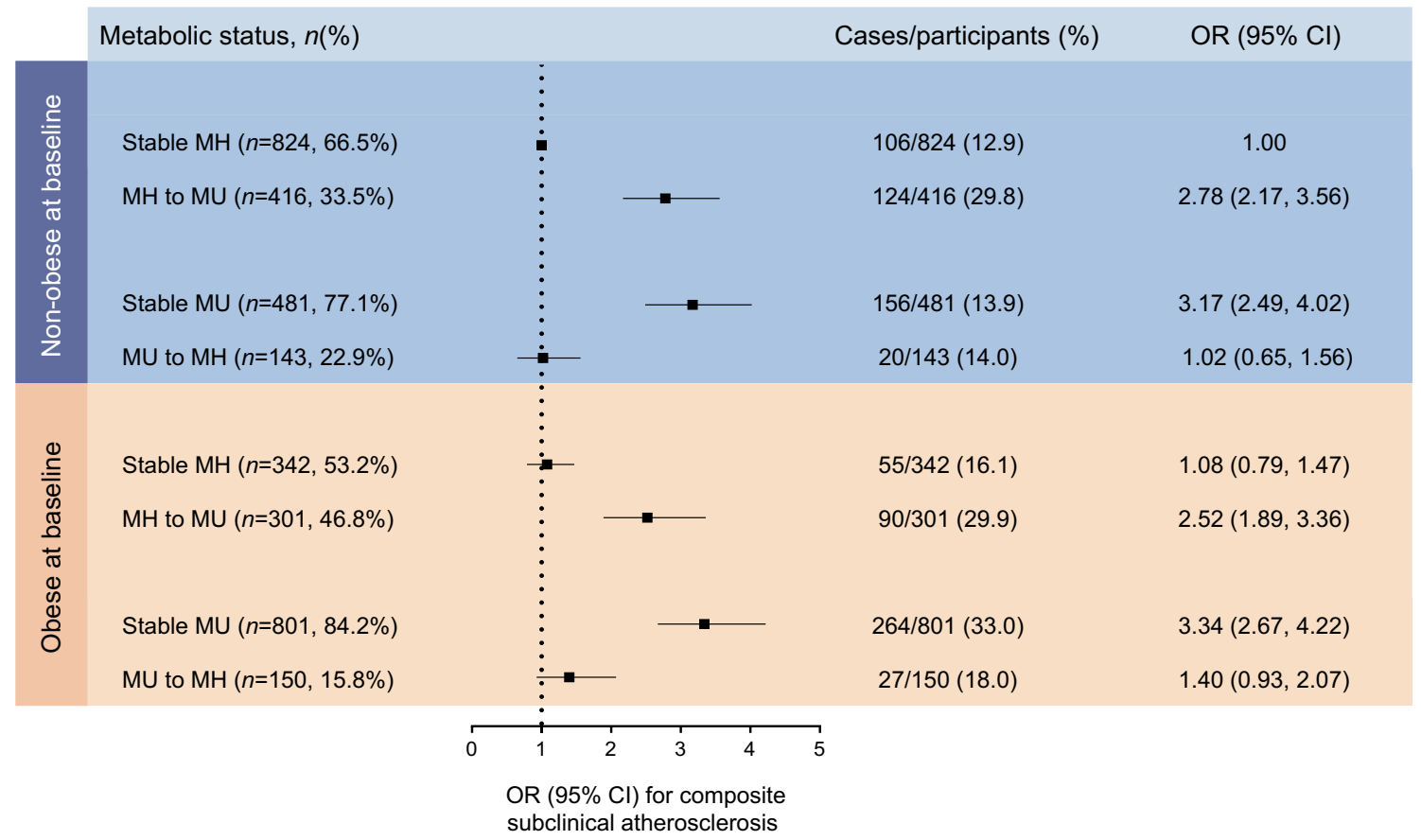

Fig. 3 ORs (95\% CIs) of composite subclinical atherosclerosis according to maintenance or transition of metabolic phenotype in non-obese and obese groups after IPW. ORs (95\% CIs) were adjusted for age, sex, current smoking (yes/no), current drinking (yes/no), education, physical activity and BMI at follow-up. MH, metabolically healthy status; MU, metabolically unhealthy status 


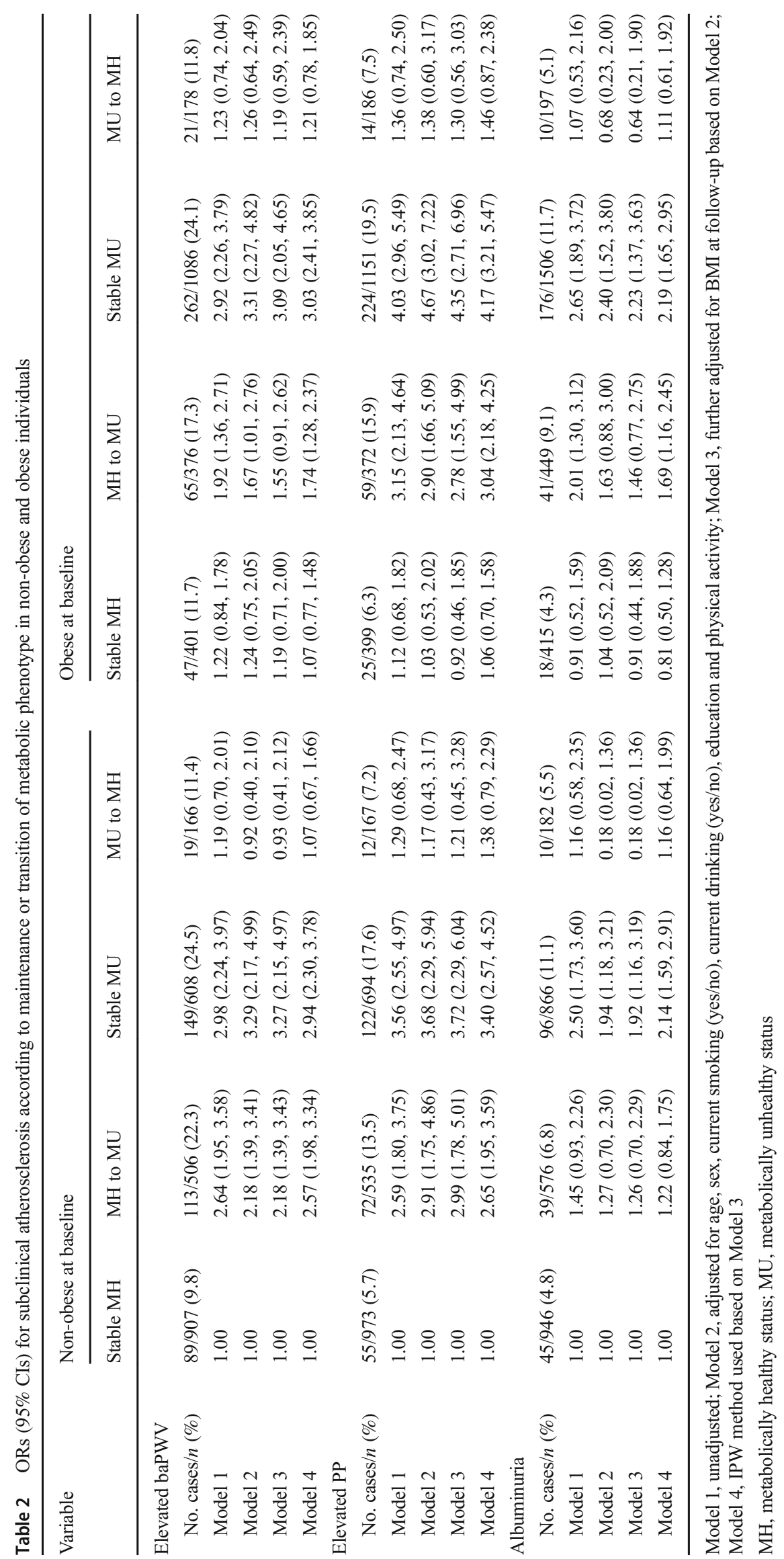



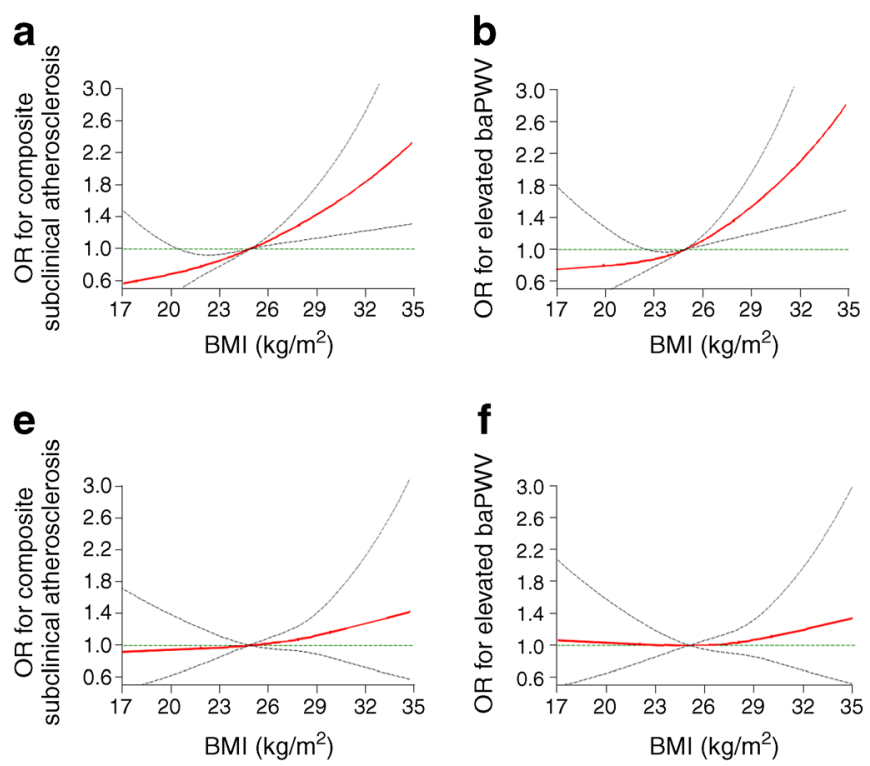

Fig. 4 Multivariate-adjusted ORs (95\% CIs) for the association of composite subclinical atherosclerosis (a, e), elevated baPWV (b, f), elevated PP (c, $\mathbf{g})$ and albuminuria (d, h) with BMI. (a-d) ORs $(95 \%$ CIs) were adjusted for age, sex, current smoking (yes/no), current

indicated that the relationship between BMI and subclinical atherosclerosis was mediated, in part, by BP (indirect effect $0.46[95 \%$ CI $0.15,0.75]$ ) and plasma glucose (indirect effect $0.12[95 \%$ CI $0.04,0.46])$.

\section{Discussion}

In this prospective study, we found that having $\mathrm{MHO}$ at baseline was not associated with an increased risk of subclinical atherosclerosis. Our data further suggested that metabolic health was a transient state. Approximately half of the individuals with MHO developed metabolically unhealthy status within the follow-up period and subsequently exhibited a higher risk of subclinical atherosclerosis. It is worth noting that the linear association between BMI and subclinical atherosclerosis was partly mediated by metabolic profiles. These findings suggest that metabolically healthy obese individuals may benefit from keeping their metabolic status stable in order to prevent subclinical atherosclerosis, and that metabolic abnormalities can increase the significance of obesity with respect to the development of subclinical atherosclerosis.

Since the concept of MHO was put forward, large-scale epidemiological studies have sought to investigate its outcome-specific prognostic value [11, 29]. The lack of a consensus on its relevance is due not only to the absence of a uniform set of criteria to define MHO but also to the fact that the literature does not consider the risk resulting from longitudinal changes in metabolic status.

The absence of the metabolic syndrome in obesity has most commonly been used to define MHO [12, 15], followed by using

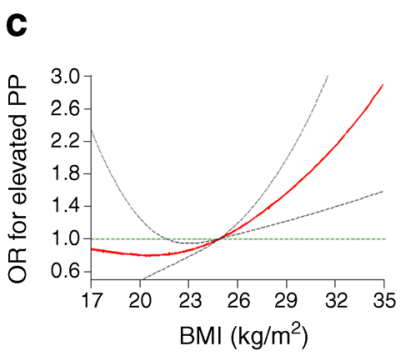

\section{d}
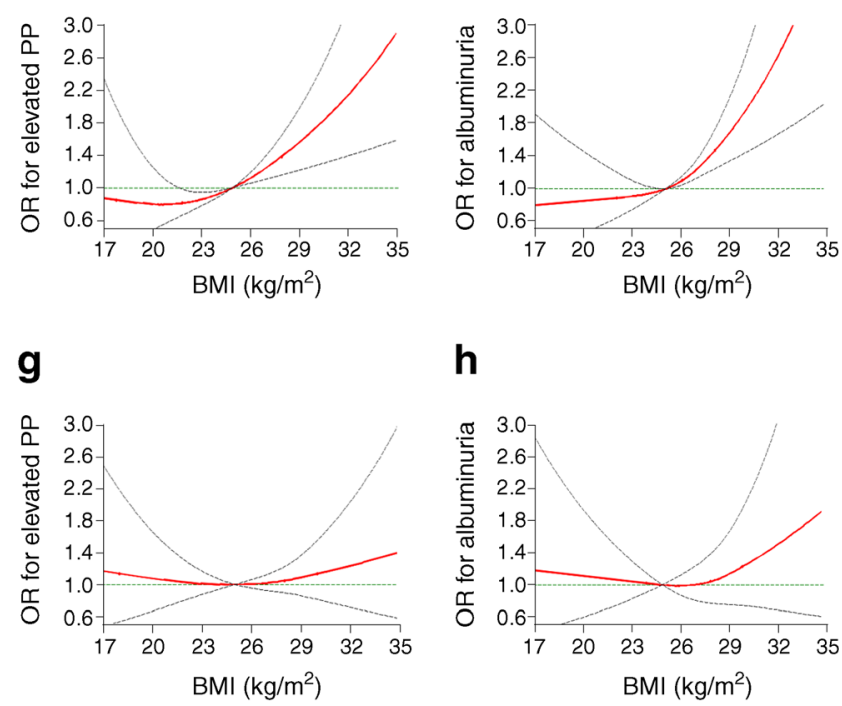

h

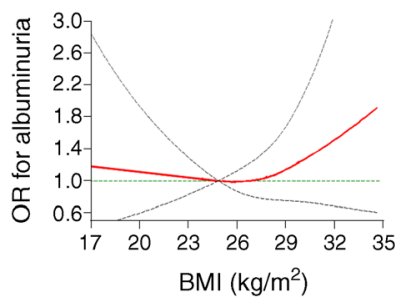

drinking (yes/no), education and physical activity. (e-h) ORs (95\% CIs) were further adjusted for FPG, triacylglycerols, systolic BP and HDLcholesterol based on (a-d). Red line, OR; dotted grey lines, 95\% CI

a HOMA-IR cut-off of 2.5 [30], or simultaneous absence of diabetes, hypertension and hyperlipidaemia [13]. Obese individuals with even two or more metabolic abnormalities could be misclassified as being 'healthy'. In the current analysis, we adopted a more rigorous definition of metabolic health, using the ATP III standard based on an established cut-off [31]. This definition ensured that relatively metabolically healthy individuals would be investigated, although an adverse effect of obesity with a metabolically benign status on incident subclinical atherosclerosis was not revealed. This supports the notion that strong additional factors play a part.

Several major meta-analyses have arrived at differing conclusions: the association of MHO phenotype with CVD incidence varies from non-significant $[14-16,32]$ to statistically significantly positive [13]. Most recently, MHO was believed to be transient in nature. In the Whitehall II cohort study, half of the initial participants with $\mathrm{MHO}$ were reported to convert to MUO within 20 years [33]. Appleton et al. documented a transition rate of $30.6 \%$ from MHO at baseline to MUO over a period of 10 years [14]. Other studies investigating the percentage of individuals transitioning from MHO to MUO over a follow-up period of 4-10 years reported the change rate to vary between $33 \%$ and $52 \%$ [13-18]. Our study demonstrated that $46.8 \%$ of individuals who initially had MHO transitioned to MUO over 4.4 years. The relatively high rate of change may be attributed to our participants being middle-aged and elderly, and hence more vulnerable to obesity and metabolic abnormalities than younger people.

Appleton et al. found no increased risk of CVD in people with persistent $\mathrm{MHO}$ or who exhibited a transition to the metabolic syndrome, which was consistent with our work [14]. A very 
recent work from MESA concluded that the transition from MHO to MUO caused a transition of CVD risk from statistical non-significance to significance [15], while the latest findings from the Nurses' Health Study highlighted that obesity remained a risk factor for CVD, irrespective of the transition of metabolic health [17].

Results of prospective studies have emphasised the role of atherosclerosis and arterial stiffness, reflected by PWV and albuminuria, as independent cardiovascular risk factors and predictors of all-cause and cardiovascular death $[34,35]$. PP is mainly related to the status of large arteries and is among the indirect rigidity estimates derived from $\mathrm{BP}$ measurements [36]. As a marker of arterial stiffness and atherosclerosis [37], PP was recently confirmed as being valuable clinically in the prediction of new-onset CVD [38]. Notably, prior work by Echouffo-Tcheugui et al. [18] using participants from the Framingham Heart Study revealed a statistically nonsignificant association between $\mathrm{MHO}$ and prevalent subclinical CVD in a cross-sectional analysis. To our knowledge, no other evidence exists regarding the risk of subclinical atherosclerosis, the early stage of CVD, in Asian individuals with stable or non-persistent MHO. In the present work, we found that having $\mathrm{MHO}$ at baseline conferred high odds of subclinical atherosclerosis on individuals who experienced a transition to metabolically unhealthy status later. The association of stable MHO with the risk of subclinical atherosclerosis stayed non-significant, when compared with persistent MHNO. Our findings strengthen the hypothesis that the initially nonsignificant risk of subclinical atherosclerosis may in fact represent a lag in risk until the transition to MUO occurs. It is anticipated that maintaining a status of $\mathrm{MHO}$ could be considered as a valid interim approach in the prevention of atherosclerosis and CVD.

Convincing evidence suggests that liver fat, which links with increased visceral adipose tissue deposition, might contribute to increased atherosclerosis and cardiometabolic risk [39]. There is a clear threshold of liver fat content (roughly $6 \%$ ) above which metabolic abnormities are fully established [40]. Metabolically healthy individuals are likely to have less visceral fat mass and lower liver fat than individuals with unhealthy metabolic status [41]. It would be interesting to further investigate whether liver fat content partly explains the deterioration of metabolic profiles and the higher risk of atherosclerosis in transient $\mathrm{MHO}$ phenotype.

Approximately one-third of the total participants were lost during follow-up, which may give rise to follow-up bias. Positively, the losses were evenly distributed with respect to the exposure status, with a dropout rate of $36.7 \%, 34.3 \%$, $31.6 \%$ and $32.9 \%$ in MHNO, MUNO, MHO and MUO groups, respectively. Furthermore, baseline characteristics were generally comparable among participants who were lost and those who continued attending the study, except for education level (data not shown). It is speculated that individuals with higher education levels might have more readily returned to the study when morbid events occurred than those with lower education levels, which possibly exaggerated the association of the exposure with atherosclerosis. Therefore, the IPW model was used to minimise the potential for follow-up bias, and the ORs for the disease did not change appreciably. Further prospective studies are warranted to test the power of replication in the validation of study results.

Considering that the metabolic status and the events were not assessed yearly, we should not ignore the possibility of reverse causality between the transition from MHO to MUO and atherosclerosis. A very recent cohort study by Zhang et al. [42] demonstrated that baseline baPWV and time-averaged PP were both positively associated with the risk of new-onset diabetes. The proposed mechanism was that the propagation of increased pressure and flow pulsations to the pancreatic bed may lead to endothelial dysfunction, which can exacerbate insulin resistance [43].

Our results also revealed a higher conversion rate from metabolic health to metabolically unhealthy status in obesity compared with normal-weight counterparts, supporting the concept that cumulative exposure to obesity is an accelerating factor for metabolic abnormalities. In addition, given that simple obesity was associated with an increased risk of subclinical atherosclerosis only after the onset of metabolic disorders, metabolic status may be a strong confounder in the obesity-atherosclerosis association. Hence, restricted cubic spline analyses were performed to demonstrate a linear association between BMI and subclinical atherosclerosis when adjusted for only age and lifestyle. The relevance was lost after further adjustment for metabolic profiles and, beyond that, metabolic disorders were the mediating factors between BMI and the outcome. Consistent with our results, a growing consensus has been reached that obesity is not an independent risk factor when obesity and metabolic disorders are taken together to evaluate the risk of atherosclerosis and CVD $[15,44]$.

This was the only study among Asians to investigate directly whether the transition of metabolic status according to BMI categories could affect the risk of subclinical atherosclerosis. However, the study has some limitations. First, the study was performed in middle-aged and elderly Chinese individuals, with a mean age of approximately 60 years, impeding drawing general conclusions. Second, the median follow-up duration of 4.4 years may have been insufficient to uncover the metabolic health transition and the incidence of events. The metabolic status and the events were not assessed yearly, thus it is unclear whether the rates of developing outcomes differ by obesity phenotype. A longer period of follow-up and multiple metabolic status and event assessments may be warranted to help clarify this issue. Third, the definition of obesity as BMI $\geq 25 \mathrm{~kg} / \mathrm{m}^{2}$ in this study was appropriate for Asians, so comparability of findings with other studies which have used the 
BMI cut-off of $30 \mathrm{~kg} / \mathrm{m}^{2}$ is limited. Finally, the variance of BMI during follow-up was not considered and this may have led to misclassification of transitions. However, this limitation was compensated for by adjusting for BMI at follow-up when assessing the association of metabolic health transitions with atherosclerosis.

In conclusion, we found that transition from $\mathrm{MHO}$ to $\mathrm{MUO}$ was associated with an increased risk of subclinical atherosclerosis. A mediation effect of metabolic profile was detected between BMI and subclinical atherosclerosis. These findings provide new evidence that persistent $\mathrm{MHO}$ can be considered as a therapeutic target in the prevention of atherosclerosis. Individuals may benefit from early behavioural and medical management to prevent the deterioration of metabolic status or to reverse mild metabolic disorders that already exist.

Acknowledgements The investigators are grateful to all participants for their cooperation in the study.

Data availability Data are available from the authors on request.

Funding This study was supported by the National Natural Science Foundation of China, (grant/award no. 81870604 and 81900741), Ministry of Science and Technology of the People's Republic of China (grant/award no. 2016YFC1304904, 2016YFC1305600 and 2016YFC1305202). YC was supported by the Shanghai Shenkang Hospital Development Center for improving the control of type 2 diabetes in the suburbs of Shanghai (16CR4020A). LL was supported by Shanghai Sailing Program (18YF1419900).

Authors' relationships and activities The authors declare that there are no relationships or activities that might bias, or be perceived to bias, their work.

Contribution statement LL, JL, YX, MX, YB, GN, WW and YC contributed to the concept and design. LL, JZ, LJ, RD, CH, TW, ML and $\mathrm{ZZ}$ contributed to the acquisition and analysis of data. LL drafted the manuscript. All authors made important contributions to editing and critically revising the manuscript for important intellectual content. All authors have approved the final version to be published. GN, WW and YC guarantee this work and have full access to all of the data and take responsibility for the integrity of the data.

\section{References}

1. Kivimäki M, Kuosma E, Ferrie JE et al (2017) Overweight, obesity, and risk of cardiometabolic multimorbidity: pooled analysis of individual-level data for 120813 adults from 16 cohort studies from the USA and Europe. Lancet Public Health 2(6):e277-e285. https:// doi.org/10.1016/S2468-2667(17)30074-9

2. Yusuf S, Hawken S, Ounpuu S et al (2004) Effect of potentially modifiable risk factors associated with myocardial infarction in 52 countries (the INTERHEART study): case-control study. Lancet 364(9438):937-952. https://doi.org/10.1016/S0140-6736(04) 17018-9

3. Stefan N, Häring HU, Schulze MB (2018) Metabolically healthy obesity: the low-hanging fruit in obesity treatment? Lancet Diabetes
Endocrinol 6(3):249-258. https://doi.org/10.1016/S2213-8587(17) 30292-9

4. Mathew H, Farr OM, Mantzoros CS (2016) Metabolic health and weight: understanding metabolically unhealthy normal weight or metabolically healthy obese patients. Metabolism 65(1):73-80. https://doi.org/10.1016/j.metabol.2015.10.019

5. Arnlöv J, Ingelsson E, Sundström J, Lind L (2010) Impact of body mass index and the metabolic syndrome on the risk of cardiovascular disease and death in middle-aged men. Circulation 121(2): 230-236. https://doi.org/10.1161/CIRCULATIONAHA.109. 887521

6. Ingelsson E, Sullivan LM, Murabito JM et al (2007) Prevalence and prognostic impact of subclinical cardiovascular disease in individuals with the metabolic syndrome and diabetes. Diabetes 56(6): 1718-1726. https://doi.org/10.2337/db07-0078

7. Di Minno MND, Di Minno A, Songia P et al (2016) Markers of subclinical atherosclerosis in patients with aortic valve sclerosis: a meta-analysis of literature studies. Int J Cardiol 223:364-370. https://doi.org/10.1016/j.ijcard.2016.08.122

8. Jørgensen L, Jenssen T, Johnsen SH (2007) Albuminuria as risk factor for initiation and progression of carotid atherosclerosis in non-diabetic persons: the Tromso study. Eur Heart J 28(3):363369. https://doi.org/10.1093/eurheartj/ehl394

9. Furtner M, Kiechl S, Mair A (2005) Urinary albumin excretion is independently associated with carotid and femoral artery atherosclerosis in the general population. Eur Heart J 26(3):279-287. https://oi.org/10.1093/eurheartj/ehi014

10. Caleyachetty R, Thomas GN, Toulis KA (2017) Metabolically healthy obese and incident cardiovascular disease events among 3.5 million men and women. J Am Coll Cardiol 70(12):14291437. https://doi.org/10.1016/j.jacc.2017.07.763

11. Kramer CK, Zinman B, Retnakaran R (2013) Are metabolically healthy overweight and obesity benign conditions? A systematic review and meta-analysis. Ann Intern Med 159(11):758-769. https://doi.org/10.7326/0003-4819-159-11-201312030-00008

12. Eckel N, Meidtner K, Kalle-Uhlmann T, Stefan N, Schulze MB (2016) Metabolically healthy obesity and cardiovascular events: a systematic review and meta-analysis. Eur J Prev Cardiol 23(9):956966. https://doi.org/10.1177/2047487315623884

13. Schulze MB (2019) Metabolic health in normal-weight and obese individuals. Diabetologia 62(4):558-566. https://doi.org/10.1007/ s00125-018-4787-8

14. Appleton SL, Seaborn CJ, Visvanathan R (2013) Diabetes and cardiovascular disease outcomes in the metabolically healthy obese phenotype: a cohort study. Diabetes Care 36(8):2388-2394. https:// doi.org/10.2337/dc12-1971

15. Mongraw-Chaffin M, Foster MC, Anderson CAM (2018) Metabolically healthy obesity, transition to metabolic syndrome, and cardiovascular risk. J Am Coll Cardiol 71(17):1857-1865. https://doi.org/10.1016/j.jacc.2018.02.055

16. Kouvari M, Panagiotakos DB, Yannakoulia M (2019) Transition from metabolically benign to metabolically unhealthy obesity and 10-year cardiovascular disease incidence: the ATTICA cohort study. Metabolism 93:18-24. https://doi.org/10.1016/j.metabol. 2019.01.003

17. Eckel N, Li Y, Kuxhaus O, Stefan N, Hu FB, Schulze MB (2018) Transition from metabolic healthy to unhealthy phenotypes and association with cardiovascular disease risk across BMI categories in 90257 women (the Nurses health study): 30 year follow-up from a prospective cohort study. Lancet Diabetes Endocrinol 6(9): 714-724. https://doi.org/10.1016/S2213-8587(18)30137-2

18. Echouffo-Tcheugui JB, Short MI, Xanthakis V et al (2019) Natural history of obesity subphenotypes: dynamic changes over two decades and prognosis in the Framingham heart study. J Clin Endocrinol Metab 104(3):738-752. https://doi.org/10.1210/jc. 2018-01321 
19. Lin L, Peng K, Du R et al (2017) High glomerular filtration rate is associated with arterial stiffness in Chinese population. J Hypertens 35(2):385-391. https://doi.org/10.1097/HJH.0000000000001158

20. Craig CL, Marshall AL, Sjöström M (2003) International physical activity questionnaire: 12-country reliability and validity. Med Sci Sports Exerc 35(8):1381-1395. https://doi.org/10.1249/01.MSS. 0000078924.61453.FB

21. Levy JC, Matthews DR, Hermans MP (1998) Correct homeostasis model assessment (HOMA) evaluation uses the computer program. Diabetes Care 21(12):2191-2192. https://doi.org/10.2337/diacare. 21.12.2191

22. WHO Expert Consultation (2004) Appropriate body-mass index for Asian populations and its implications for policy and intervention strategies. Lancet 363(9403):157-163. https://doi.org/10.1016/ S0140-6736(03)15268-3

23. Hamaguchi M, Kojima T, Takeda N et al (2005) The metabolic syndrome as a predictor of nonalcoholic fatty liver disease. Ann Intern Med 143(10):722-728. https://doi.org/10.7326/0003-4819143-10-200511150-00009

24. Lin L, Peng K, Du R et al (2017) Metabolically healthy obesity and incident chronic kidney disease: the role of systemic inflammation in a prospective study. Obesity (Silver Spring) 25(3):634-641. https://doi.org/10.1002/oby.21768

25. Hinnouho GM, Czernichow S, Singh-Manoux A (2013) Metabolically healthy obesity and risk of mortality: does the definition of metabolic health matter? Diabetes Care 36(8):2294-2300. https://doi.org/10.2337/dc14-0101

26. Huang $\mathrm{Y}, \mathrm{Bi} \mathrm{Y}, \mathrm{Xu} \mathrm{M}$ et al (2012) Nonalcoholic fatty liver disease is associated with atherosclerosis in middle-aged and elderly Chinese. Arterioscler Thromb Vasc Biol 32(9):2321-2326. https://doi.org/ 10.1161/ATVBAHA.112.252957

27. Heerspink HJL, Greene T, Tighiouart H et al (2019) Change in albuminuria as a surrogate endpoint for progression of kidney disease: a meta-analysis of treatment effects in randomised clinical trials. Lancet Diabetes Endocrinol 7(2):128-139. https://doi.org/10. 1016/S2213-8587(18)30314-0

28. Durrleman S, Simon R (1989) Flexible regression models with cubic splines. Stat Med 8(5):551-561

29. Kaur A, Johnston DG, Godsland IF (2016) Does metabolic health in overweight and obesity persist? - individual variation and cardiovascular mortality over two decades. Eur J Endocrinol 175(2):133143. https://doi.org/10.1530/EJE-16-0095

30. Lu J, Bi Y, Wang T et al (2014) The relationship between insulinsensitive obesity and cardiovascular diseases in a Chinese population: results of the REACTION study. Int J Cardiol 172(2):388394. https://doi.org/10.1016/j.ijcard.2014.01.073

31. Jung CH, Lee MJ, Kang YM et al (2015) The risk of incident type 2 diabetes in a Korean metabolically healthy obese population: the role of systemic inflammation. J Clin Endocrinol Metab 100(3): 934-941. https://doi.org/10.1210/jc.2014-3885

32. Hamer M, Stamatakis E (2012) Metabolically healthy obesity and risk of all-cause and cardiovascular disease mortality. J Clin Endocrinol Metab 97(7):2482-2488. https://doi.org/10.1210/jc. 2011-3475

33. Bell JA, Hamer M, Sabia S, Singh-Manoux A, Batty GD, Kivimaki M (2015) The natural course of healthy obesity over 20 years. J Am
Coll Cardiol 65(1):101-102. https://doi.org/10.1016/j.jacc.2014. 09.077

34. Vlachopoulos C, Aznaouridis K, Stefanadis C (2010) Prediction of cardiovascular events and all-cause mortality with arterial stiffness: a systematic review and meta-analysis. J Am Coll Cardiol 55(13): 1318-1327. https://doi.org/10.1016/j.jacc.2009.10.061

35. Chronic Kidney Disease Prognosis Consortium, Matsushita K, van der Velde M et al (2010) Association of estimated glomerular filtration rate and albuminuria with all-cause and cardiovascular mortality in general population cohorts: a collaborative meta-analysis. Lancet 375(9731):2073-2081. https://doi.org/10.1016/S01406736(10)60674-5

36. Briet M, Boutouyrie P, Laurent S, London GM (2012) Arterial stiffness and pulse pressure in CKD and ESRD. Kidney Int 82(4): 388-400. https://doi.org/10.1038/ki.2012.131

37. Ferreira I, Hovind P, Schalkwijk CG, Parving HH, Stehouwer CDA, Rossing P (2018) Biomarkers of inflammation and endothelial dysfunction as predictors of pulse pressure and incident hypertension in type 1 diabetes: a 20-year life-course study in an inception cohort. Diabetologia 61(1):231-241. https://doi.org/10.1007/ s00125-017-4470-5

38. Said MA, Eppinga RN, Lipsic E, Verweij N, van der Harst P (2018) Relationship of arterial stiffness index and pulse pressure with cardiovascular disease and mortality. J Am Heart Assoc 7(2): e007621. https://doi.org/10.1161/JAHA.117.007621

39. Neeland IJ, Ross R, Després JP et al (2019) Visceral and ectopic fat, atherosclerosis, and cardiometabolic disease: a position statement. Lancet Diabetes Endocrinol 7(9):715-725. https://doi.org/10.1016/ S2213-8587(19)30084-1

40. Bril F, Barb D, Portillo-Sanchez P et al (2017) Metabolic and histological implications of intrahepatic triglyceride content in nonalcoholic fatty liver disease. Hepatology 65(4):1132-1144. https://doi. org/10.1002/hep.28985

41. Stefan N, Häring HU, Hu FB, Schulze MB (2013) Metabolically healthy obesity: epidemiology, mechanisms, and clinical implications. Lancet Diabetes Endocrinol 1(2):152-162. https://doi.org/10. 1016/S2213-8587(13)70062-7

42. Zhang Y, He P, Li Y et al (2019) Positive association between baseline brachial-ankle pulse wave velocity and the risk of newonset diabetes in hypertensive patients. Cardiovasc Diabetol 18(1): 111. https://doi.org/10.1186/s12933-019-0915-0

43. Balletshofer BM, Rittig K, Enderle MD et al (2000) Endothelial dysfunction is detectable in young normotensive first-degree relatives of individuals with type 2 diabetes in association with insulin resistance. Circulation 101(15):1780-1784. https://doi.org/10. 1161/01.CIR.101.15.1780

44. Fan J, Song Y, Chen Y, Hui R, Zhang W (2013) Combined effect of obesity and cardio-metabolic abnormality on the risk of cardiovascular disease: a meta-analysis of prospective cohort studies. Int J Cardiol 168(5):4761-4768. https://doi.org/10.1016/j.ijcard.2013. 07.230

Publisher's note Springer Nature remains neutral with regard to jurisdictional claims in published maps and institutional affiliations. 